\title{
Tau positron emission tomography, cerebrospinal fluid and plasma biomarkers of neurodegeneration, and neurocognitive testing: an exploratory study of participants with myotonic dystrophy type 1
}

\author{
Robert Jr Laforce ${ }^{1}$ (D) Caroline Dallaire-Théroux ${ }^{1} \cdot$ Annie M. Racine ${ }^{2} \cdot$ Gersham Dent $^{2} \cdot$ Cristian Salinas-Valenzuela $^{2}$. \\ Elizabeth Poulin ${ }^{1} \cdot$ Anne-Marie Cayer ${ }^{1} \cdot$ Daphnée Bédard-Tremblay $^{1} \cdot$ Thierry Rouleau-Bonenfant $^{1}$. \\ Frédéric St-Onge $^{1}$ · Susanna Schraen-Maschke ${ }^{3,4}$. Jean-Mathieu Beauregard ${ }^{1} \cdot$ Nicolas Sergeant $^{3,4}$ • Jack Puymirat ${ }^{1}$
}

Received: 2 November 2021 / Revised: 11 January 2022 / Accepted: 11 January 2022 / Published online: 1 February 2022

(c) The Author(s) 2022

\begin{abstract}
Objective To investigate Tau pathology using multimodal biomarkers of neurodegeneration and neurocognition in participants with myotonic dystrophy type 1 (DM1).

Methods We recruited twelve participants with DM1 and, for comparison, two participants with Alzheimer's Disease (AD). Participants underwent cognitive screening and social cognition testing using the Dépistage Cognitif de Québec (DCQ), among other tests. Biomarkers included Tau PET with [18F]-AV-1451, CSF (A $\beta$, Tau, phospho-Tau), and plasma (A $\beta$, Tau, Nf-L, GFAP) studies.

Results Of the twelve DM1 participants, seven completed the full protocol (Neurocognition 11/12; PET 7/12, CSF 9/12, plasma 12/12). Three DM1 participants were cognitively impaired (CI). On average, CI DM1 participants had lower scores on the DCQ compared to cognitively unimpaired (CU) DM1 participants (75.5/100 vs. 91.4/100) and were older (54 vs. 44 years old) but did not differ in years of education (11.3 vs. 11.1). The majority (6/7) of DM1 participants had no appreciable PET signal. Only one of the CI participants presented with elevated Tau PET SUVR in bilateral medial temporal lobes. This participant was the eldest and most cognitively impaired, and had the lowest CSF A $\beta$ 1-42 and the highest CSF Tau levels, all suggestive of co-existing AD. CSF Tau and phospho-Tau levels were higher in the $3 \mathrm{CI}$ compared to CU DM1 participants, but with a mean value lower than that typically observed in AD. Nf-L and GFAP were elevated in most DM1 participants (9/11 and 8/11, respectively). Finally, CSF phospho-Tau was significantly correlated with plasma Nf-L concentrations.

Conclusions and relevance We observed heterogenous cognitive and biomarker profiles in individuals with DM1. While some participants presented with abnormal PET and/or CSF Tau, these patterns were highly variable and only present in a small subset. Although DM1 may indeed represent a non-AD Tauopathy, the Tau-PET tracer used in this study was unable to detect an in vivo Tau DM1 signature in this small cohort. Interestingly, most DM1 participants presented with elevated plasma Nf-L and GFAP levels, suggestive of other, possibly related, central brain alterations which motivate further research. This pioneering study provides novel insights towards the potential relationship between biomarkers and neurocognitive deficits commonly seen in DM1.
\end{abstract}

Keywords Tau PET $\cdot$ Imaging $\cdot \mathrm{CSF} \cdot$ Cognition $\cdot$ Social cognition $\cdot$ Myotonic dystrophy type 1

Nicolas Sergeant and Jack Puymirat have contributed equally to the present work and share last authorship.

Robert Jr Laforce

robert.laforce@fmed.ulaval.ca

Extended author information available on the last page of the article

\section{Introduction}

Myotonic dystrophy type 1 (DM1) is a chronic, multisystemic, autosomal dominant neuromuscular disorder caused by an expanded CTG repeat in the DMPK gene. It is the most common form of adult muscular dystrophy worldwide. Neurologically, DM1 can manifest as muscle weakness, mild intellectual disability, and neurobehavioral changes with impairments in social cognition [19, 25, 32]. Underlying pathophysiological mechanisms 
are unknown but several neuropathological reports suggest that DM1 may be a Tauopathy where hyper and abnormally phosphorylated microtubule-associated Tau proteins accumulate in a topographic distribution similar to early stages of Alzheimer's disease (AD) $[2,15]$. Others have reported that brain Tau is alternatively spliced in DM1 [7, 11, 24] and CSF Tau levels may be modestly elevated [21,31]. As reported, if Tau is mis-spliced and elevated in the CSF, it could be a pertinent biomarker for brain dysfunction and disease progression, with potential to demonstrate therapeutic benefit in clinical trials.

Structural neuroimaging abnormalities are well documented in prior studies of DM1 participants, including widespread gray matter loss in cortical and subcortical structures, ventriculomegaly, and white matter lesions [8]. White matter abnormalities [27], focal hypoperfusion [22] and reduced glucose metabolism in frontal, parietal and temporal lobes [20] have also been detected using diffusion tensor imaging, SPECT and FDG-PET. By contrast, there are no prior reports investigating Tau pathology in vivo in the brains of DM1 participants despite the fact that various Tau PET ligands are now readily available. One of the most studied Tau PET tracers, [18F]-AV1451, was employed for this study. While many studies support the use of [18F]-AV-1451 to detect Tau pathology in participants with $\mathrm{AD}$, its value for detecting non-AD Tauopathies is much less understood [12, 26]. DM1 is the only known disease characterized by the preferential aggregation of $0 \mathrm{~N} 3 \mathrm{R}$ Tau isoforms [2], and whether [18F]-AV-1451 binds to the conformation of Tau observed in DM1 [7, 28] is not yet known. A few studies have examined CSF biomarkers of neurodegeneration in DM1 and have reported fairly similar results. For instance, one study reported a statistically significant decrease in CSF A $\beta$ 1-42 concentrations and increased levels of t-Tau in DM1 participants compared to healthy controls [31]. Similarly, while no statistically significant differences were reported, Peric et al. [21] showed that $t$-Tau and $p$-Tau levels were numerically higher and $A \beta$ 1-42 was numerically lower in DM1 participants compared to healthy controls.

The goal of this exploratory study was thus to investigate Tau pathology in vivo in a small but well-characterized sample of DM1 participants using several biomarkers of neurodegeneration, including [18F]-AV-1451 Tau PET imaging, CSF and plasma biomarkers. We further aimed to study the relationship between pathology-associated biomarkers and severity of neurocognitive impairments in DM1, including social cognition deficits.

\section{Methods}

\section{Participants}

We initially recruited 19 participants with a genetic diagnosis of DM1 and two with Alzheimer's disease (AD) from neurology clinics at the largest academic tertiary care center in Eastern Quebec City, QC, Canada. The two participants with AD served as quality controls for Tau PET. Seven of the 19 DM1 participants withdrew from the study (3/7 changed their mind upon signing the informed consent, 2/7 were scheduled for surgery, and $2 / 7$ were lost to follow-up); therefore, the final analysis sample reported herein included 12 DM1 participants and 2 AD participants. All participants provided written informed consent. This study was approved by the local Research Ethics Board.

\section{Neurocognitive and social cognition testing}

Participants underwent cognitive screening using the Dépistage Cognitif de Québec ${ }^{20}$ (DCQ; http://dcqtest.org/), the Montreal Cognitive Assessment [18] (MoCA) and the Mini-Mental State Examination [6] (MMSE world and 100-7 versions). The DCQ is a 25-min cognitive screening test composed of five indexes (Memory, Visuospatial, Executive, Language, and Behavioral). It has been validated in both healthy participants and patients with atypical dementias. Assessment of social cognition was performed using the Behavioral Index of the DCQ. The Clinical Dementia Rating [17] (CDR) was used to grade the relative severity of dementia with scores from 0 (no impairment) to 3 (severe impairment). Participants were designated as cognitively impaired (CI) if their performance fell 2 standard deviations below the mean of the normative sample either on the MoCA or DCQ based on a separate, previously analyzed, normative sample [9]; those not meeting that criterion were considered to be cognitively unimpaired (CU).

\section{Tau PET imaging}

During the PET scanning visit, each participant received an intravenous bolus infusion of $\sim 10 \mathrm{mCi}$ of $\left[{ }^{18} \mathrm{~F}\right]-\mathrm{AV}-1451$, a radioligand which preferentially binds to neurofibrillary tangles. Sixty minutes post administration of the radioligand, participants were scanned for $30 \mathrm{~min}$. Images were reconstructed applying scatter and attenuation correction and decay corrected to time of radioligand administration. Reconstructed images were also corrected for motion that might have occurred during scanning. Concomitant to the PET scan, participants also underwent a structural T1 MRI scan for the purpose of PET registration and region of interest (ROI) analysis. Standardized uptake value ratios (SUVR) images were generated using superior-cropped cerebellar gray matter as the reference region and average SUVR values were calculated in key ROIs, including composite ROIs corresponding to Braak I-II, Braak III-IV, and Braak V-VI regions $[1,13,23]$. 


\section{CSF biomarkers}

CSF collection was performed through a lumbar puncture by an experienced neurologist. CSF concentrations of $A \beta$ 1-42, A $\beta$ 1-40, total-Tau, and phospho-Tau at threonine 181 were measured with the automated chemiluminescent enzyme-immunoassay (Lumipulse G 1200, Fujirebio Europe, Gent, Belgium). Analysis of the quality controls provided in the kits including three different levels of concentrations for each biomarker showed $\mathrm{CV}<5 \%$ (2.3\%, $4.8 \%, 5.0 \%$ and $4.0 \%$, respectively). Standard CSF cut-offs provided by the manufacturer for distinguishing $\mathrm{AD}$ from other dementia and controls were $56.6 \mathrm{pg} / \mathrm{mL}$ for phosphoTau, $400 \mathrm{pg} / \mathrm{mL}$ for total-Tau, $600 \mathrm{pg} / \mathrm{mL}$ for $\mathrm{A} \beta 1-42$ and $6.9 \%$ for $A \beta 1-42 / A \beta 1-40$ ratio [4].

\section{Plasma biomarkers}

Blood samples were drawn before the lumbar puncture for quantification of plasma levels of amyloid $A \beta 1-42$ and $A \beta$ 1-40, total Tau, Glial Fibrillary Acidic Protein (GFAP) and Neurofilament Light Chain (Nf-L). Plasma A $\beta$ peptide assay was performed using the INNO-BIA kit (Fujirebio Europe NV, formely Innogenetics NV, Belgium), based on a multiplex xMAP technique with a LABScan-200 system (Luminex BV, The Netherlands). The interserial CV of $A \beta 1-40$ was between 7.1 and $7.6 \%$ (for levels of 216 and $106 \mathrm{pg} / \mathrm{mL}$, respectively) and the interserial CV of $A \beta 1-42$ was between 3.0 and $9.4 \%$ (for levels of 93 and $198 \mathrm{pg} /$ $\mathrm{mL}$, respectively). The Neurology-4-plex assay was used (Lot 501858, Simoa technology, Quanterix Corporation, Lexington, MA, USA) on the SIMOA platform (Simoa technology, Quanterix Corporation, Lexington, MA, USA) to measure Nf-L, total-Tau and GFAP in plasma samples. This plasma biomarker analysis was performed by SpotToLab thanks to the clinical proteomic platform of Montpelier CHU headed by Professor Christophe HIRTZ (c-hirtz@chu-montpellier.fr). The limit of quantification for Nf-L was $0.38 \mathrm{pg} / \mathrm{mL}$, total-Tau was $0.10 \mathrm{pg} / \mathrm{mL}$ and GFAP was $0.923 \mathrm{pg} / \mathrm{mL}$. Analysis of the quality controls provided with the kit included low and high concentrations of Nf-L, total-Tau and GFAP with a CV lower than $10 \%$ ( $4 \%, 10 \%$ and $6 \%$, respectively). To minimize the matrix effects, all samples were diluted fourfold with the diluent provided in the kit (phosphate buffer with bovine serum and heterophilic blocker solution) before analysis. Median plasma values of ten healthy donors indicated in the data sheet of the kit were: $8.58 \mathrm{pg} / \mathrm{mL}$ for Nf-L, $64.2 \mathrm{pg} / \mathrm{mL}$ for GFAP and $2.31 \mathrm{pg} / \mathrm{mL}$ for total-Tau, respectively; these were used as reference levels for our analysis to indicate abnormality.

\section{Statistical analyses}

Descriptive results are expressed as means $\pm \mathrm{SD}$ as well as the median and interval between the 1st and 4th quartile. Although this study is exploratory and primarily hypothesis generating, we performed preliminary analyses to compare biomarker profiles between CI vs. CU DM1 participants and to investigate associations between biomarker modalities. First, we performed uncorrected $t$ tests on CSF and plasma biomarker levels between $\mathrm{CI}$ and $\mathrm{CU}$ participants. Second, we examined correlations between CSF and plasma biomarkers through Spearman rank correlation matrix using Graphpad Prism Software 8.4.2; correlations are represented on a heatmap. Spearman correlations between CSF and plasma biomarker levels were also explored (Stata SE 15.1). $P$ values $<0.05$ were considered significant.

\section{Data availability}

Anonymized data will be made available upon any reasonable request from qualified investigators.

\section{Results}

\section{Neurocognition and social cognition}

Cognitive and social cognition testing scores are provided in Table 1, along with demographic information of the 12 DM1 participants and 2 AD participants. The two AD participants (\#1 and \#2) displayed a classic amnestic AD presentation on cognitive testing (MMSE and MoCA) with impaired episodic memory, visuospatial and executive skills.

Table 1 Demographics of the study participants

\begin{tabular}{lll}
\hline Variables & DM1 $(n=12)$ & $\begin{array}{l}\text { Alzheimer's } \\
\text { disease } \\
(n=2)^{*}\end{array}$ \\
\hline Age (years; mean, sd) & $47(11.2)$ & 67 \\
Gender (F/M) & $3 / 8$ & $1 / 1$ \\
Education (years; mean, sd) & $11.2(1.2)$ & 12 \\
MMSE world (/30; mean, sd) & $28.8(1.9)$ & 21 \\
MMSE 100-7 (/30; mean, sd) & $28.4(2.1)$ & 17 \\
MoCA (/30; mean, sd) & $26.6(3.6)$ & 13.5 \\
DCQ Total (/100; mean, sd) & $87.0(9.9)$ & N/A \\
DCQ Behavioral Index (/20; mean, sd) & $17.8(3.0)$ & N/A \\
CDR (mean, sd) & $0.3(0.6)$ & 2 \\
\hline
\end{tabular}

MMSE Mini-Mental State Examination, MoCA Montreal Cognitive Assessment, DCQ Dépistage Cognitif de Québec (http://dcqtest.org/), $C S F$ cerebrospinal fluid, CDR Clinical Dementia Rating

*Standard deviations are not reported for AD participants given sample size of two 
As expected, the CDR was higher for the two AD participants compared to the DM1 participants, suggesting greater functional impairment. Three (3/12) DM1 participants (\#3, $\# 8$ and \#19) were CI, as evidenced by lower mean scores on the MMSE (28/30 vs. $29 / 30)$, DCQ (79/100 vs. $90 / 100)$ and/ or MoCA (23.3/30 vs. 29/30) relative to normative data previously reported [9]. Their performance on both the MMSE and MoCA indicated executive dysfunctions and the Social Cognition Index of the DCQ indicated significant social deficits, both defined by scores which fell 2 standard deviations below the mean of the normative sample. More specifically, three of the five indexes of the DCQ were impaired (Executive Index, Visuospatial Index, Social Cognition Index). CI participants were older than CU participants ( 53 vs. 46 years on average) but did not differ in number of years of education. Finally, there were no correlations between cognitive performance and blood repeat length.

\section{Tau PET imaging biomarkers}

The two AD participants (\#1 and \#2) showed a typical AD-like pattern on Tau PET, including high retention across frontal, parietal, and temporal cortices and the medial temporal lobe; e.g. SUVR $\geq 1.5$ (average of left and right) across composite ROIs corresponding to Braak stages I-V (see Fig. 1 and [Supplementary] Table S1). Of the three CI participants, one (\#19) presented with increased Tau PET signal bilaterally in the medial temporal lobes (e.g. amygdala and hippocampus; Fig. 1). There was also prominent signal in striatum, but this is most likely attributable to off-target binding due to AV-1451 non-specificity [10]. SUVR values for this participant in the Braak I-II composite were 1.36 (right) and 1.50 (left), nearing that seen in the two AD cases ([Supplementary] Table S1). Patient \#19 (aged 69 yo) was also the most severely cognitively impaired of the DM1 participants examined $(\mathrm{DCQ}=61.5 / 100 ; \mathrm{MoCA}=17 / 30)$. Patient \#8 (aged 45 yo), who was also mildly cognitively impaired $(\mathrm{DCQ}=82.5 / 100 ; \mathrm{MoCA}=30 / 30)$, showed slight focal increase in Tau PET signal (e.g. right Braak I-II SUVR $=1.30$ ). Tau PET in the last CI participant (\#3) was unremarkable (see Fig. 1) despite mild cognitive deficits $(\mathrm{DCQ}=82.5 / 100 ; \mathrm{MoCA}=27 / 30)$. The CU participants showed no uptake beyond that seen in the cerebellar reference region. Due to the limited signal present, statistical analysis comparing Tau PET SUVR between CI and CU participants was not performed.

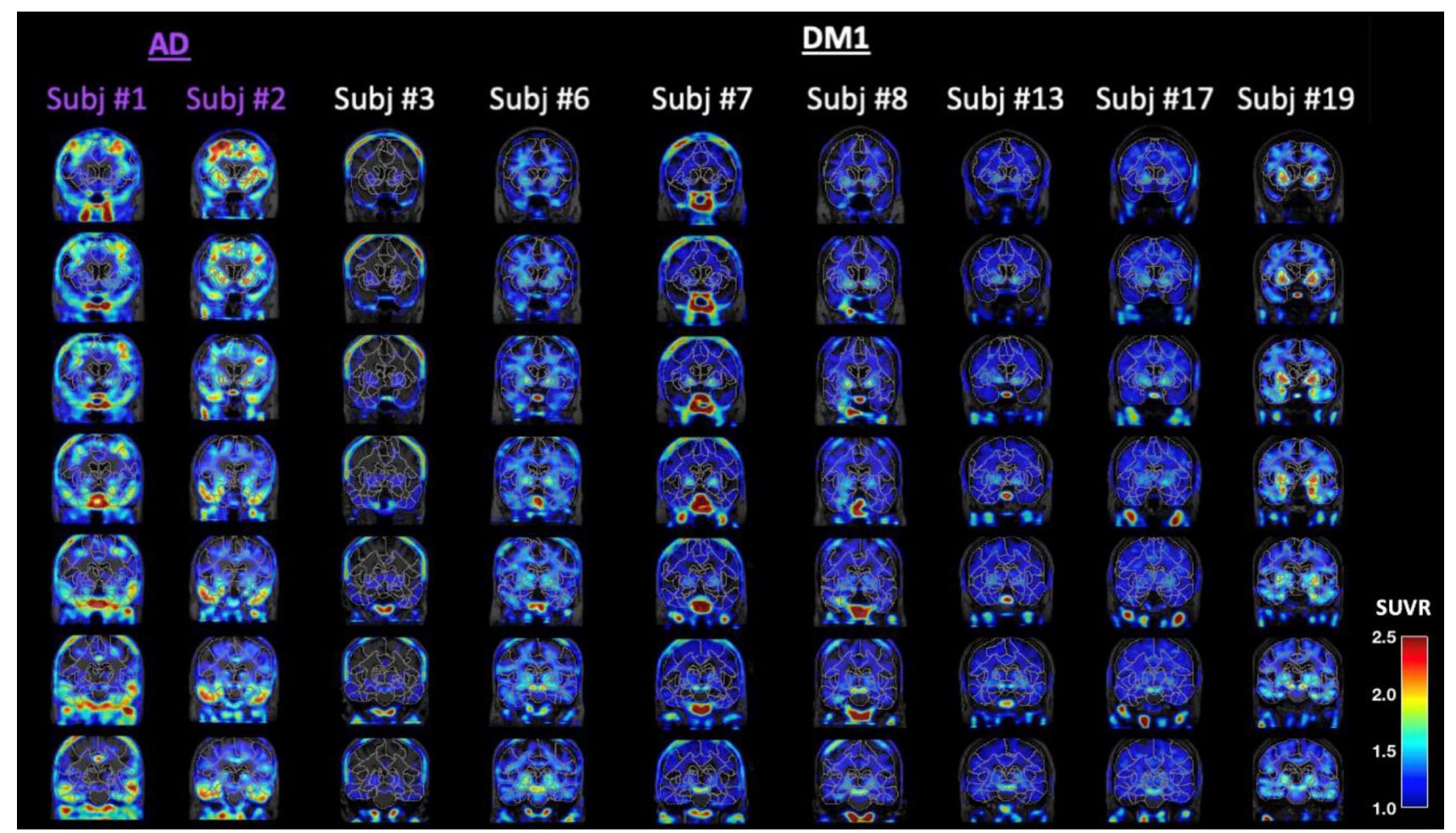

Fig. 1 MK-6240 Tau PET images of AD (purple) and DM1 (white) participants. Coronal slices are displayed progressing anterior to posterior. SUVR images are overlaid on subject-specific T1 MRI images, as well as the outline of the subject-specific ROI atlas parcellation. SUVR scale is from 1 to 2.5 


\section{CSF biomarkers}

Nine (9/12) DM1 participants completed the CSF portion of the study (see Table 2). CSF Total-Tau and phosphoTau average concentrations were $256.44 \pm 244 \mathrm{ng} / \mathrm{mL}$ and $38.88 \pm 24.2 \mathrm{ng} / \mathrm{mL}$, respectively. These concentrations were below the cut-off thresholds for an AD-like profile (i.e. $>400 \mathrm{pg} / \mathrm{mL}$ for total-Tau and $>56.6 \mathrm{pg} / \mathrm{mL}$ for phospho-Tau). Interestingly, CSF total-Tau and phospho-Tau levels were on average higher in CI participants (\#3, \#8 and \#19) compared to CU participants (423 vs. $151.6 \mathrm{pg} / \mathrm{mL}$ and 64.3 vs. $26.42 \mathrm{pg} / \mathrm{mL}$ on average, respectively). Notably, patient \#19 had the highest CSF total-Tau and phospho-Tau concentrations.

Average CSF concentration of $A \beta 1=42, A \beta 1-40$ and $A \beta$ 1-42/A $\beta 1-40$ ratio in this DM1 series were $609.78 \pm 270 \mathrm{ng} /$ $\mathrm{mL}, 7791.67 \pm 2311 \mathrm{ng} / \mathrm{mL}$ and $8 \% \pm 2 \%$, respectively. Therefore, on average, A $\beta$ 1-42 did not reach the threshold for what is considered AD pathological levels $(<600 \mathrm{pg} /$ $\mathrm{mL}$ ) while $\mathrm{A} \beta$ 1-42/A $\beta$ 1-40 ratio was slightly above an $\mathrm{AD}$ threshold $(>6.9 \%)$. The latter appears to be driven by relatively low levels of $A \beta 1-40$, which reflects overall production of amyloid peptides. Together, these data do not support presence of AD-type central amyloidopathy in DM1. When comparing DM1 CI participants to the CU DM1 participants, CSF amyloid markers $\mathrm{A} \beta$ 1-42 and $\mathrm{A} \beta$ 1-42/A $\beta$ 1-40 ratio were lower in CI (553 vs. $638 \mathrm{pg} / \mathrm{mL}$ and 6.0 vs. $8.7 \%$ respectively), although this appears to be driven by DM1 patient \#19. The mean level of A $\beta$ 1-40 was higher in CI participants compared to CU participants (9060 vs. $7129 \mathrm{pg} /$ $\mathrm{mL})$.

\section{Plasma Biomarkers}

All twelve DM1 participants completed the plasma portion of the study (see Table 3). Plasma biomarkers of neurodegeneration (Nf-L and Tau) and of glial cell lesions (GFAP) were higher in CI participants than CU participants (Nf-L: 50.91 vs. $13.04 \mathrm{pg} / \mathrm{mL}$; Tau: 2.87 vs. $1.59 \mathrm{pg} / \mathrm{mL}$; GFAP: 94.78 vs. $81.95 \mathrm{pg} / \mathrm{mL}$ ). Of note, plasma Nf-L was markedly higher in patient \#19 compared to all other participants (Table 3). Plasma A $\beta$ 1-42/A $\beta$ 1-40 ratio was also higher in CI participants than in CU participants ( 0.224 vs. $0.193 \mathrm{pg} /$ $\mathrm{mL}$ ). The A $\beta$ 1-40 plasma concentrations were similar between $\mathrm{CI}$ and $\mathrm{CU}$ while $\mathrm{A} \beta$ 1-42 plasma concentrations were higher in CI (46.9 pg/mL) versus CU (38.48 pg/mL).

\section{Correlations between CSF and plasma biomarkers}

Correlations were performed using the non-parametric Spearman rank correlation matrix as the cohort is small and the data do not follow a Gaussian distribution (see Fig. 2). Plasma Nf-L concentrations were shown to significantly correlate with CSF phospho-Tau concentration $(r=0.90 ; p=0.005)$. Of note, this association remained significant even after removing participant \#19, who had
Table 2 CSF biomarkers in DM1 participants

\begin{tabular}{|c|c|c|c|c|c|}
\hline Samples & $\mathrm{A} \beta 1-42(\mathrm{pg} / \mathrm{mL})$ & $\mathrm{A} \beta 1-40(\mathrm{pg} / \mathrm{mL})$ & $\begin{array}{l}\mathrm{A} \beta \text { 1-42/A } \beta \\
1-40 \text { Ratio }(\%)\end{array}$ & Total-Tau (pg/mL) & $\begin{array}{l}\text { Phospho- } \\
\text { Tau (pg/ } \\
\text { mL) }\end{array}$ \\
\hline \#3 & 675 & 9897 & $6.8 \%$ & 444 & 61.3 \\
\hline \#4 & 1158 & 11,874 & $9.8 \%$ & 283 & 53.4 \\
\hline \#6 & N/A & N/A & N/A & N/A & N/A \\
\hline$\# 7$ & 510 & 5742 & $8.9 \%$ & 135 & 21.7 \\
\hline \#8 & 762 & 9136 & $8.3 \%$ & 244 & 38.8 \\
\hline$\# 10$ & 635 & 7298 & $8.7 \%$ & 111 & 20.1 \\
\hline$\# 12$ & 496 & 5744 & $8.6 \%$ & 281 & 24.2 \\
\hline \#13 & N/A & N/A & N/A & N/A & N/A \\
\hline$\# 14$ & N/A & N/A & N/A & N/A & N/A \\
\hline \#15 & 335 & 4463 & $7.5 \%$ & 115 & 17.4 \\
\hline \#17 & 694 & 7823 & $8.9 \%$ & 114 & 19.5 \\
\hline \#19 & 223 & 8148 & $2.7 \%$ & 581 & 92.8 \\
\hline Mean & 609.78 & 7791.67 & $8 \%$ & 256.44 & 38.80 \\
\hline Median & 635.00 & 7823.00 & $9 \%$ & 244.00 & 24.20 \\
\hline SD & 270.12 & 2311.68 & $2 \%$ & 165.14 & 25.79 \\
\hline 1st quartile & 496.00 & 5744.00 & $8 \%$ & 115.00 & 20.10 \\
\hline Last quartile & 694.00 & 8148.00 & $9 \%$ & 281.00 & 38.80 \\
\hline
\end{tabular}

Standard CSF cut-offs provided by the manufacturer for distinguishing Alzheimer's disease from other dementia and controls are $56.6 \mathrm{pg} / \mathrm{mL}$ for phospho-Tau, $400 \mathrm{pg} / \mathrm{mL}$ for total-Tau, $600 \mathrm{pg} / \mathrm{mL}$ for A $\beta 1-42$ and $6.9 \%$ for $\mathrm{A} \beta$ 1-42/A $\beta$ 1-40 ratio 
Table 3 Plasma biomarkers in DM1 participants

\begin{tabular}{|c|c|c|c|c|c|c|}
\hline Participant samples & GFAP (pg/mL) & NF-L (pg/mL) & TAU $(\mathrm{pg} / \mathrm{mL})$ & $\mathrm{A} \beta 1-42(\mathrm{pg} / \mathrm{mL})$ & $\mathrm{A} \beta 1-40(\mathrm{pg} / \mathrm{mL})$ & $\begin{array}{l}\mathrm{A} \beta \text { 1-42/A } \beta \\
1-40 \text { Ratio }\end{array}$ \\
\hline \#3 & 99.75 & 39.08 & 3.78 & 51.6 & 242.7 & 0.213 \\
\hline$\# 4$ & 70.43 & 15.54 & 0.47 & 48.3 & 198.0 & 0.244 \\
\hline \#6 & 61.42 & 20.84 & 1.40 & 58.5 & 267.3 & 0.219 \\
\hline$\# 7$ & 52.15 & 13.69 & 2.63 & 16.2 & 260.7 & 0.062 \\
\hline$\# 8$ & 92.61 & 12.80 & 2.89 & 42.6 & 173.1 & 0.246 \\
\hline$\# 10$ & 113.05 & 14.77 & 0.84 & 54.0 & 212.1 & 0.255 \\
\hline$\# 12$ & N/A & N/A & N/A & 49.5 & 193.5 & 0.256 \\
\hline$\# 13$ & 48.50 & 4.86 & 1.08 & 31.2 & 160.8 & 0.194 \\
\hline$\# 14$ & 122.42 & 20.18 & 3.12 & 42.0 & 188.1 & 0.223 \\
\hline$\# 15$ & 81.50 & 5.70 & 1.56 & 23.1 & 204.0 & 0.113 \\
\hline$\# 17$ & 106.17 & 8.72 & 1.60 & 34.5 & 192.9 & 0.179 \\
\hline \#19 & 91.99 & 100.86 & 1.95 & 46.5 & 216.6 & 0.215 \\
\hline Mean & 85.45 & 23.37 & 1.94 & 41.50 & 209.15 & 0.20 \\
\hline Median & 91.99 & 14.77 & 1.60 & 44.55 & 201.00 & 0.22 \\
\hline SD & 24.80 & 27.35 & 1.04 & 12.85 & 32.99 & 0.06 \\
\hline 1st quartile & 65.92 & 10.76 & 1.24 & 33.68 & 191.70 & 0.19 \\
\hline Last quartile & 99.39 & 20.51 & 2.29 & 48.60 & 213.23 & 0.24 \\
\hline
\end{tabular}

For reference, median plasma values of ten healthy donors indicated in the data sheet of the kit were: $8.58 \mathrm{pg} / \mathrm{mL}$ for Nf-L, $64.2 \mathrm{pg} / \mathrm{mL}$ for GFAP and $2.31 \mathrm{pg} / \mathrm{mL}$ for total-Tau, respectively



Fig. 2 Correlations between CSF and plasma quantifications

the highest levels of both biomarkers $(r=0.86, p=0.024)$. Although the associations did not reach statistical significance, plasma Nf-L was also highly correlated with CSF total-Tau $(r=0.71)$ and with CSF A $\beta 1-40(r=0.60)$. Correlations between Tau PET and fluid biomarkers were not performed due to low SUVR signal in all but one patient.

\section{Discussion}

To our knowledge, this is the first study to explore Tau pathology in vivo in DM1 using Tau PET, CSF, plasma and neurocognitive biomarkers. Overall, the patterns and relationships between cognitive and biomarker profiles were heterogenous. Nevertheless, this study adds to a growing body of literature investigating the possible presence of Tau pathology in DM1. It further provides critical insight on sensitivity of tools to detect Tau pathology in vivo in these participants.

Three (3/12) DM1 participants were cognitively impaired, as evidenced by lower average scores on the DCQ and/or MoCA. Their neuropsychological profile indicated primary deficits in executive function and social cognition. Out of the three CI participants, two presented with some level of elevated Tau PET signal in the temporal lobes. The magnitude and extent of Tau PET signal was lower than that typically seen in AD participants, and only strongly present in one of the DM1 participants (\#19). While this is consistent with the topological distribution of Tau pathology in neuropathological examination of post-mortem brain of DM1 participants $[5,7,15]$, it also aligns with a typical early ADlike pattern with initial Tau deposits in the medial temporal lobes. The other DM1 participants did not show notably increased Tau PET signal relative to the reference region. Prior studies have suggested that $[18 \mathrm{~F}]-\mathrm{AV}-1451$ specifically binds to $3 \mathrm{R} / 4 \mathrm{R}$ paired helical Tauopathy characteristic of 
$\mathrm{AD}$, with relatively low affinity for other Tau filaments [12, 26]; to our knowledge, no studies have specifically tested whether 18F-AV-1451 or other available Tau PET tracers bind to the specific Tau conformation (principally made of 0N3R Tau isoforms) found in DM1 [2, 28]. Based on the current data showing little to no binding of [18F]-AV-1451 in the majority of DM1 participants in this sample, and only strong binding in the patient with possible concomitant $\mathrm{AD}$, we conjecture that $[18 \mathrm{~F}]-\mathrm{AV}-1451$ either does not bind to DM1-specific Tau conformations, or that it is not sufficiently sensitive to detect the low levels of Tau pathology that are present in many or most DM1 participants. It is also possible that sub-populations of DM1 participants with higher levels of Tau pathology with inclusions made of both 3R and $4 \mathrm{R}$ Tau isoforms [7] that can be detected by [18-F]-AV1451 exist, but additional studies with larger populations are needed to test that empirically. The potential value of other Tau PET tracers with different selectivity or specificity for DM1-specific Tau remains to be determined.

Relationships between CSF Tau and phospho-Tau with cognitive impairment were similarly heterogeneous. Two of the three CI DM1 participants (participants \#3 and \# 19) had elevated CSF total Tau and phospho-Tau above the AD cutoff levels. Levels of CSF Tau and phospho-Tau biomarkers were higher in participants that were $\mathrm{CI}$ than $\mathrm{CU}$, but with a mean value lower than that typically observed in $\mathrm{AD}$ and more similar to other Tauopathies such as frontotemporal lobar degeneration [16]. In contrast to the CSF Tau findings, CSF amyloid levels were more homogenous. Apart from one patient (\#19), all DM1 participants had normal CSF $A \beta$ 1-42/A $\beta$ 1-40 ratio levels suggesting the absence of AD-type central amyloidopathy as reported previously [15]. If post-mortem analysis of patient \#19 were to confirm a diagnosis of concomitant $\mathrm{AD}$, it is mostly likely that their abnormal CSF amyloid pattern is attributable to AD pathophysiology rather than DM1. At least two other studies have previously analyzed CSF amyloid and Tau levels in DM1 participants [21, 31]. In both studies Tau CSF levels in DM1 were greater than in healthy controls. Curiously, both studies also observed lower levels of CSF A $\beta$ 1-42 in DM1 compared to controls; however, they did not analyze $A \beta$ 1-42/A $1-\beta 40$ ratio, which is considered a more reliable measure of cerebral amyloidopathy [30]. This requires further replication given that amyloid deposits are seldom associated to neurofibrillary degeneration in DM1 brains [7, 15].

Plasma biomarkers of neurodegeneration (Nf-L and Tau), glial cell lesions (GFAP), and amyloid (A $\beta$ 1-42, A $\beta$ 1-40, and $\mathrm{A} \beta$ 1-42/A $\beta$ 1-40 ratio) were all higher in CI participants than CU participants in this sample. Plasma Nf-L levels and GFAP levels were increased in most participants (8/10 and $7 / 10$, respectively) relative to median levels in a healthy control sample, indicating that neuronal and glial alterations are possibly common to most DM1 participants; however, lack of a control sample in this study limits our interpretation. The gliosis in DM1 brain remains ill-defined [29]; increased levels of plasma GFAP could reflect an elevated gliosis process in DM1, but this requires further confirmation by post-mortem correlative analyses. Plasma Nf-L was strongly correlated with CSF phospho-Tau. A recent study [3] observed a similar correlation between plasma Nf-L and CSF Total-tau and phospho-Tau in cognitively normal participants with AD pathology defined by an AD CSF profile, while another study [14] did not. To our knowledge, this is the first study to investigate these fluid biomarker associations in DM1 participants. Correlation between CSF pTau and plasma Nf-L may reflect increased neuronal injury in both $\mathrm{AD}$ and $\mathrm{DM} 1$ but post-mortem analyses and replication in other DM1 cohorts are necessary to further corroborate this hypothesis.

This novel study adds to a growing body of research investigating central nervous system abnormalities in DM1, including Tau pathology. While some participants presented with abnormal Tau measured by Tau PET and/or CSF, these patterns were highly variable and only present in a small subset of our sample. Interestingly, the patient with the most severe cognitive impairment in our sample also demonstrated the most elevated Tau PET signal, the highest CSF phospho-Tau and Tau levels, and lowest CSF A $\beta$ 1-42 and $A \beta$ 1-42/ $A \beta$ 1-40 levels, consistent with a typical $A D$ biochemical profile. Whether this biomarker signal is related to more severe $\mathrm{DM} 1$ pathology or concomitant $\mathrm{AD}$ remains unknown and will necessitate further investigation. Indeed, one major limitation of this study is lack of post-mortem data to confirm biomarker sensitivity to DM1 Tau pathology, and to rule out other comorbidities, like $\mathrm{AD}$, which may be contributing to both the observed cognitive impairment and abnormal Tau and/or amyloid biomarkers. A second major limitation is the small sample size; however, this study was designed to be exploratory and hypothesis generating. Postmortem neuropathological examination (e.g., with autoradiographical confirmation of Tau-PET binding in DM1 brain tissue) and studies with larger samples of DM1 participants are necessary to further evaluate whether other Tau PET tracers and/or fluid biomarkers could represent pathological biomarkers in DM1. Studying various Tauopathies using multimodal biomarkers will help elucidate the pathological mechanisms of Tau and may help identify valuable biomarkers to support future therapies and clinical trials.

Author contributions RL acted as PI, performed the lumbar punctures and wrote the core of the paper. CD-T wrote a draft of the introduction, reviewed the final version, recruited participants and tested them. A-MC, DB-T, TR-B, FSt-O recruited participants, scheduled and tested them. EP helped in analyzing the Tau PET data and brain imaging files. J-MB acted as expert in nuclear medicine and facilitated Tau PET protocols. JP helped recruiting DM1 participants from his neurology clinics. The Biogen team (AR, CS-V, GD) conducted Tau PET analyze 
and contributed to manuscript writing and revision. The University of Lille, Inserm and CHRU of Lille team (SS-M and NS), conducted CSF and plasma analyses, statistical analyses and co-wrote the manuscript.

Funding This study was possible as a result of a grant from AFM Telethon to Robert Laforce, Jack Puymirat and Nicolas Sergeant.

\section{Declarations}

Conflicts of interest The first and last authors have no disclosure.

Ethical standard This research was approved by the local Research Ethics Committee.

Open Access This article is licensed under a Creative Commons Attribution 4.0 International License, which permits use, sharing, adaptation, distribution and reproduction in any medium or format, as long as you give appropriate credit to the original author(s) and the source, provide a link to the Creative Commons licence, and indicate if changes were made. The images or other third party material in this article are included in the article's Creative Commons licence, unless indicated otherwise in a credit line to the material. If material is not included in the article's Creative Commons licence and your intended use is not permitted by statutory regulation or exceeds the permitted use, you will need to obtain permission directly from the copyright holder. To view a copy of this licence, visit http://creativecommons.org/licenses/by/4.0/.

\section{References}

1. Braak H, Braak E (1991) Neuropathological stageing of Alzheimer-related changes. Acta Neuropathol 82:239-259

2. Caillet-Boudin ML, Fernandez-Gomez FJ, Tran H, Dhaenens CM, Buee L, Sergeant N (2014) Brain pathology in myotonic dystrophy: when tauopathy meets spliceopathy and RNAopathy. Front Mol Neurosci 6:57

3. Clark C, Lewczuk P, Kornhuber J, Richiardi J, Marechal B, Karikari TK, Blennow K, Zetterberg H, Popp J (2021) Plasma neurofilament light and phosphorylated tau 181 as biomarkers of Alzheimer's disease pathology and clinical disease progression. Alzheimers Res Ther 13:65

4. Dakterzada F, Lopez-Ortega R, Arias A, Riba-Llena I, RuizJulian M, Huerto R, Tahan N, Pinol-Ripoll G (2021) Assessment of the concordance and diagnostic accuracy between elecsys and lumipulse fully automated platforms and innotest. Front Aging Neurosci 13:604119

5. Fernandez-Gomez F, Tran H, Dhaenens CM, Caillet-Boudin ML, Schraen-Maschke S, Blum D, Sablonniere B, Buee-Scherrer V, Buee L, Sergeant N (2019) Myotonic dystrophy: an RNA toxic gain of function tauopathy? Adv Exp Med Biol 1184:207-216

6. Folstein MF, Folstein SE, McHugh PR (1975) "Mini-mental state". A practical method for grading the cognitive state of patients for the clinician. J Psychiatr Res 12:189-198

7. Jimenez-Marin A, Diez I, Labayru G, Sistiaga A, Caballero MC, Andres-Benito P, Sepulcre J, Ferrer I, Lopez de Munain A, Cortes JM (2021) Transcriptional signatures of synaptic vesicle genes define myotonic dystrophy type I neurodegeneration. Neuropathol Appl Neurobiol. https://doi.org/10.1111/nan.12725

8. Labayru G, Diez I, Sepulcre J, Fernandez E, Zulaica M, Cortes JM, Lopez de Munain A, Sistiaga A (2019) Regional brain atrophy in gray and white matter is associated with cognitive impairment in Myotonic Dystrophy type 1. Neuroimage Clin 24:102078
9. Laforce R Jr, Sellami L, Bergeron D, Paradis A, Verret L, Fortin MP, Houde M, Roy M, Poulin S, Macoir J, Hudon C, Bouchard RW (2018) Validation of the Depistage Cognitif de Quebec: a new cognitive screening tool for atypical dementias. Arch Clin Neuropsychol 33:57-65

10. Laforce R Jr, Soucy JP, Sellami L, Dallaire-Theroux C, Brunet F, Bergeron D, Miller BL, Ossenkoppele R (2018) Molecular imaging in dementia: past, present, and future. Alzheimers Dement $14: 1522-1552$

11. Leroy O, Wang J, Maurage CA, Parent M, Cooper T, Buee L, Sergeant N, Andreadis A, Caillet-Boudin ML (2006) Brain-specific change in alternative splicing of Tau exon 6 in myotonic dystrophy type 1. Biochim Biophys Acta 1762:460-467

12. Leuzy A, Chiotis K, Lemoine L, Gillberg PG, Almkvist O, Rodriguez-Vieitez E, Nordberg A (2019) Tau PET imaging in neurodegenerative tauopathies-still a challenge. Mol Psychiatry 24:1112-1134

13. Maass A, Landau S, Baker SL, Horng A, Lockhart SN, La Joie R, Rabinovici GD, Jagust WJ, Alzheimer's Disease Neuroimaging I (2017) Comparison of multiple tau-PET measures as biomarkers in aging and Alzheimer's disease. Neuroimage 157:448-463

14. Mattsson N, Zetterberg H, Janelidze S, Insel PS, Andreasson U, Stomrud E, Palmqvist S, Baker D, Tan Hehir CA, Jeromin A, Hanlon D, Song L, Shaw LM, Trojanowski JQ, Weiner MW, Hansson O, Blennow K, Investigators A (2016) Plasma tau in Alzheimer disease. Neurology 87:1827-1835

15. Maurage CA, Udd B, Ruchoux MM, Vermersch P, Kalimo H, Krahe R, Delacourte A, Sergeant N (2005) Similar brain tau pathology in DM2/PROMM and DM1/Steinert disease. Neurology 65:1636-1638

16. Meeter LHH, Vijverberg EG, Del Campo M, Rozemuller AJM, Donker Kaat L, de Jong FJ, van der Flier WM, Teunissen CE, van Swieten JC, Pijnenburg YAL (2018) Clinical value of neurofilament and phospho-tau/tau ratio in the frontotemporal dementia spectrum. Neurology 90:e1231-e1239

17. Morris JC (1993) The clinical dementia rating (CDR): current version and scoring rules. Neurology 43:2412-2414

18. Nasreddine ZS, Phillips NA, Bedirian V, Charbonneau S, Whitehead V, Collin I, Cummings JL, Chertkow H (2005) The Montreal Cognitive Assessment, MoCA: a brief screening tool for mild cognitive impairment. J Am Geriatr Soc 53:695-699

19. Okkersen K, Buskes M, Groenewoud J, Kessels RPC, Knoop H, van Engelen B, Raaphorst J (2017) The cognitive profile of myotonic dystrophy type 1: a systematic review and meta-analysis. Cortex 95:143-155

20. Peric S, Brajkovic L, Belanovic B, Ilic V, Salak-Djokic B, Basta I, Rakocevic Stojanovic V (2017) Brain positron emission tomography in patients with myotonic dystrophy type 1 and type 2 . J Neurol Sci 378:187-192

21. Peric S, Mandic-Stojmenovic G, Markovic I, Stefanova E, Ilic V, Parojcic A, Misirlic-Dencic S, Ostojic M, Rakocevic-Stojanovic V, Kostic V (2014) Cerebrospinal fluid biomarkers of neurodegeneration in patients with juvenile and classic myotonic dystrophy type 1. Eur J Neurol 21:231-237

22. Romeo V, Pegoraro E, Ferrati C, Squarzanti F, Soraru G, Palmieri A, Zucchetta P, Antunovic L, Bonifazi E, Novelli G, Trevisan CP, Ermani M, Manara R, Angelini C (2010) Brain involvement in myotonic dystrophies: neuroimaging and neuropsychological comparative study in DM1 and DM2. J Neurol 257:1246-1255

23. Scholl M, Lockhart SN, Schonhaut DR, O'Neil JP, Janabi M, Ossenkoppele R, Baker SL, Vogel JW, Faria J, Schwimmer HD, Rabinovici GD, Jagust WJ (2016) PET Imaging of Tau deposition in the aging human brain. Neuron 89:971-982

24. Sergeant N, Sablonniere B, Schraen-Maschke S, Ghestem A, Maurage CA, Wattez A, Vermersch P, Delacourte A (2001) 
Dysregulation of human brain microtubule-associated tau mRNA maturation in myotonic dystrophy type 1 . Hum Mol Genet 10:2143-2155

25. Serra L, Bianchi G, Bruschini M, Giulietti G, Domenico CD, Bonarota S, Petrucci A, Silvestri G, Perna A, Meola G, Caltagirone C, Bozzali M (2020) Abnormal cortical thickness is associated with deficits in social cognition in patients with myotonic dystrophy type 1. Front Neurol 11:113

26. Soleimani-Meigooni DN, Iaccarino L, La Joie R, Baker S, Bourakova V, Boxer AL, Edwards L, Eser R, Gorno-Tempini ML, Jagust WJ, Janabi M, Kramer JH, Lesman-Segev OH, Mellinger T, Miller BL, Pham J, Rosen HJ, Spina S, Seeley WW, Strom A, Grinberg LT, Rabinovici GD (2020) 18F-flortaucipir PET to autopsy comparisons in Alzheimer's disease and other neurodegenerative diseases. Brain 143:3477-3494

27. van Dorst M, Okkersen K, Kessels RPC, Meijer FJA, Monckton DG, van Engelen BGM, Tuladhar AM, Raaphorst J, Consortium O (2019) Structural white matter networks in myotonic dystrophy type 1. Neuroimage Clin 21:101615
28. Vermersch P, Sergeant N, Ruchoux MM, Hofmann-Radvanyi H, Wattez A, Petit H, Dwailly P, Delacourte A (1996) Specific tau variants in the brains of patients with myotonic dystrophy. Neurology 47:711-717

29. Weijs R, Okkersen K, van Engelen B, Kusters B, Lammens M, Aronica E, Raaphorst J, van Walsum AMVC (2021) Human brain pathology in myotonic dystrophy type 1: a systematic review. Neuropathology 41:3-20

30. Wiltfang J, Lewczuk P, Otto M (2016) Biomarkers for dementia and other neurodegenerative diseases: current developments. Nervenarzt 87:1305-1309

31. Winblad S, Mansson JE, Blennow K, Jensen C, Samuelsson L, Lindberg C (2008) Cerebrospinal fluid tau and amyloid beta42 protein in patients with myotonic dystrophy type 1. Eur J Neurol 15:947-952

32. Winblad S, Samuelsson L, Lindberg C, Meola G (2016) Cognition in myotonic dystrophy type 1: a 5-year follow-up study. Eur J Neurol 23:1471-1476

\title{
Authors and Affiliations
}

\author{
Robert Jr Laforce ${ }^{1}$ (D) Caroline Dallaire-Théroux ${ }^{1} \cdot$ Annie M. Racine $^{2} \cdot$ Gersham Dent $^{2} \cdot$ Cristian Salinas-Valenzuela $^{2}$. \\ Elizabeth Poulin ${ }^{1}$. Anne-Marie Cayer ${ }^{1}$. Daphnée Bédard-Tremblay ${ }^{1}$ - Thierry Rouleau-Bonenfant ${ }^{1}$. \\ Frédéric St-Onge ${ }^{1}$. Susanna Schraen-Maschke ${ }^{3,4}$ - Jean-Mathieu Beauregard ${ }^{1}$. Nicolas Sergeant ${ }^{3,4}$ - Jack Puymirat ${ }^{1}$ \\ 1 Clinique Interdisciplinaire de Mémoire, CHU de Québec, \\ Québec, QC, Canada \\ 2 Biogen Inc, Cambridge, MA, USA \\ 3 Université de Lille, Inserm UMRS1172, CHU Lille, Lille, \\ France \\ 4 Alzheimer \& Tauopathies, LabEx DISTALZ, Lille, France
}

